\title{
ASYMPTOTIC WAVE-LIKE MODELING OF DIELECTRIC LENSES
}

\author{
${ }^{1}$ Yurchenko V. B. and ${ }^{2}$ Itintas A. A. \\ ${ }^{1}$ Experimental Physics Dept., National University of Ireland, Maynooth, Co. Kildare, \\ Ireland \\ E-mail: v.yurchenko@nuim.ie \\ ${ }^{2}$ Electrical and Electronics Engineering Department, Bilkent University, Ankara, \\ Turkey \\ E-mail: altintas@ee.bilkent.edu.tr
}

\begin{abstract}
We propose asymptotic wave-like approximations for the accurate modeling of dielectric lenses used in quasi-optical systems of millimeter, submillimeter, and infrared wave applications. For the comparison, we obtain an exact full-wave solution of two-dimensional focusing lens problem and use it as a benchmark for testing and validation of asymptotic models being proposed.
\end{abstract}

Keywords: Dielectric, lens, microwave, infrared, THz, high frequency, asymptotic, full wave, physical optics, simulation, modeling.

\section{INTRODUCTION}

Accurate design of large-scale quasi-optical systems with dielectric lenses requires advanced wave-like asymptotic modeling of refractive components (lenses) similar to physical optics modeling of reflector antennas.

An example of the system this kind is QUaD, a sub-millimeter-wave telescope for Cosmic Microwave Polarization experiment on DESI site at South Pole $[1,2]$. For the high precision instrument to be obtained, an extremely accurate modeling of aberration effects introduced by the telescope optics is needed. The problem is particularly acute when the fine structure of radiation field in the focal domain has to be found or polarization effects to be computed with high accuracy. In this case, the problem requires advanced methods for the wave-like asymptotic modeling of lenses analogous to physical optics modeling of large reflectors [3].

When developing methods for the accurate asymptotic modeling, comparisons with exact solutions are of great importance. In the meantime, exact simulation of electromagnetic wave scattering by a dielectric body is a complicated problem, especially, for systems of large electrical size as compared to the radiation wavelength.

There are various approaches to numerical solution of dielectric scattering problems ranging from the finite difference, finite elements and similar methods [4] to special sophisticated formulations based on the integral equations $[5,6]$ combined with regularization techniques of their solution [7-9]. Exact methods are usually limited to systems of small size, often less than ten wavelengths in diameter even in those cases when advanced solvers are available (e.g., Ansoft HFSS software). Besides, there is limited number of exact solutions for dielectric lenses described in the literature.

One example is a recent simulation of a convex micro-cylindrical axial-lens [10] by using the integral equation method [6] with no regularization. A rigorous solution based on regularization technique is presented in $[8,9]$ for a special class of small-size twodimensional problems of relatively complicated geometry. A promising hybrid method is proposed for diffractive micro-lenses in [11] where the finite difference method solves the problem in the near field of the lens and the partial plane wave propagation is used to transform the solution to the far-field domain.

In general, however, there are no exact solutions published for the canonical lens geometries, e.g., for the common two-sided convex lenses of conventional shape. Similarly, there are no advanced asymptotic methods developed for these problems to be accurate and efficient for exceeding the capabilities of conventional ray tracing approach. Meanwhile, it is this kind of problems that needs both the full-wave solution and the enhanced asymptotic modeling in practical applications.

In this paper, we develop a few wave-like asymptotic simulation methods and compare them with fullwave analytic solutions obtained for the problem of electromagnetic wave focusing with two-dimensional (2D) convex dielectric lenses of conventional shape when the lens cross-section profile is formed by two circular arcs as shown in Fig. 1.

First, we obtain a full-wave analytic solution to the problem of plane wave focusing by a $2 \mathrm{D}$ dielectric 
lens and compute the wave fields in the focal domain of the lens for two orthogonal polarizations, TM and TE, of the incident wave, respectively.

Then, we propose a few asymptotic methods analogous to the physical optics approach with the aim to reproduce the exact solutions with sufficient accuracy for dielectric lenses of relatively large size at various values of the lens parameters.

\section{Full-Wave Simulations of DIELECTRIC LENSES}

\subsection{Simulation Method}

Full-wave analytic solutions for dielectric lenses can be obtained by direct methods when using cylindrical wave expansions of the incident and scattered fields with respect to $\mathrm{O} 1$ and $\mathrm{O} 2$ frames associated with the circular arcs S1L and S2L that form the lens crosssection profile (Fig. 1).

The Neumann addition theorem for cylindrical functions is used for transforming the expansions from one frame to another when satisfying the boundary conditions for tangential field components at the lens surfaces. Because of rapid growth or decay of cylindrical functions with the angular harmonic index, direct solutions of this kind are limited to lenses of relatively small size.

To apply the method to lenses of moderate size, we run numerical computations with quadruple precision arithmetic. This allows us to simulate lenses with the surface curvature radii $R 1, R 2$ and the lens diameter $D$ exceeding $10 \lambda$ when having the lens refractive index $n=1.5$ ( $\lambda$ is the free-space wavelength). Further increase of the lens size can be achieved with regularization methods.

\subsection{Simulation Results}

The results showing the wave focusing effects in both the $\mathrm{E}$ (TM) and H (TE) polarizations are plotted in Fig. 2. For convenience, we choose the lens refractive index $n=1.5$ so that the geometrical focal point F1

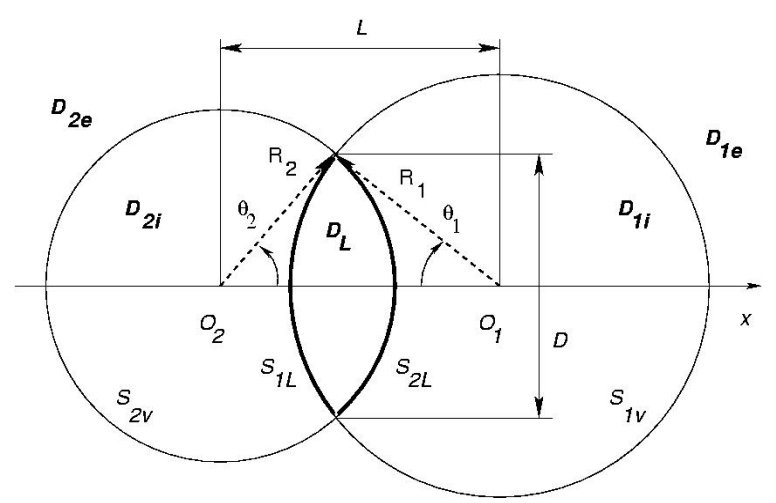

Fig. 1. Geometry of a 2D convex dielectric lens (cross-section profile). The excitation plane wave of either TM $(\mathrm{E} \| \mathrm{Oz})$ or TE $(\mathrm{H} \| \mathrm{Oz})$ polarization is assumed to be incident from the left.

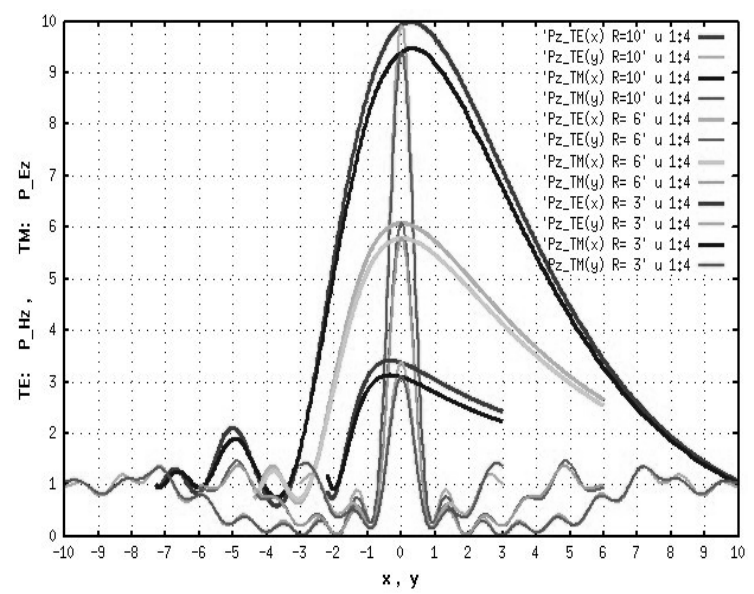

Fig. 2. Exact solutions for the TE, TM plane wave incidence on 2D dielectric lenses: transverse (y) and longitudinal $(x)$ cuts of $H_{z}^{2}$ (TE) and $E_{z}^{2}$ (TM) power flux components via $\mathrm{O}_{1}$ focal point $\left(n=1.5, f=R_{1}=R_{2}=D, D=\{3,6\right.$, 10\} $\left.\lambda, \theta_{1}=\theta_{2}=30^{\circ}\right)$.

(F2) coincides with the frame origin $\mathrm{O} 1$ (O2) when the excitation is due to the plane wave incident from the left (right).

Power flux densities PE and PH associated with the $\mathrm{Ez}$ and $\mathrm{Hz}$ field components of the TM and TE waves, respectively, are shown in the focal domain of the lens in transverse (y) and longitudinal (x) cuts passing through the focal point $\mathrm{F} 1=\mathrm{O} 1$ (the total power flux of each wave is about two times greater for each curve, respectively).

A common feature observed in all simulations is that the TE polarized wave produces greater energy density at the focal point as compared to the TM wave of the same incident power. This is the result of difference in TE and TM wave transmission at oblique incidence at the lens surfaces as expressed by the Fresnel coefficients.

The second feature is that the width of the focal spot defined as the distance between the first minima of the filed is twice the wavelength. In the meantime, the width of diffraction fringes is one wavelength for lenses of different size, with nearly identical patterns in both polarizations.

In addition, there are some intrinsic resonant effects associated with lenses, which are especially pronounced in the narrow-band applications. With increasing the size of lens and the bandwidth of radiation, the resonant effects smear out and become insignificant.

\section{Asymptotic Wave-Like Modeling OF DIELECTRIC LENSES}

\subsection{TWO-TERM KIRCHHOFF APPROXIMATION}

Wave-like asymptotic approximations for dielectric lenses proposed in this work are based on various modifications of diffraction integrals that represent 
wave propagation from the lens exit surface (surface S2L in Fig. 1 for the excitation wave incident from the left) to the observation points behind the lens (e.g., in the domain around the focal point $\mathrm{O} 1$ ).

Propagation of the incident wave from the entry surface S1L to the exit surface S2L can be evaluated by ray tracing through the lens body with account of (a) the transmission Fresnel coefficients at S1L and S2L surfaces, (b) phase increments along the rays inside the lens and (c) wave amplitude increments (decrements) due to the ray convergence (divergence) because of refraction. This evaluation is rather accurate for typical lenses because of relatively short ray propagation path inside the lens as compared to the lens transverse dimensions.

The first method we consider is based on the twoterm Kirchhoff diffraction integral. The integral is evaluated over the extended exit surface S2 that consists of the lens exit surface S2L and the free-space surface S2F extended from the lens rim to infinity in transverse direction.

The first term in this integral depends on the wave amplitude $U$ ( $E z$ or $H z$, depending on polarization) that is directly evaluated at the exit surface S2 as explained above. The second term depends, however, on the normal derivative of $U$ at $\mathrm{S} 2$ that has to be approximated by using the $U$ values at $\mathrm{S} 2$ and the set of ray directions.

Depending on particular details of this approximation, we obtain slightly different simulation models, though, typically, they are rather similar in final results (we denote this approach as KU model).

\subsection{ONE-TERM RAYLEIGH-SOMMERFELD FORMULATION MODIFIED FOR CURVED SURFACES}

Another asymptotic model we obtain by starting with one-term Rayleigh-Sommerfeld diffraction integral and modifying it for non-planar (curved) exit surfaces.

Since the idea of the original Rayleigh-Sommerfeld formulation is the choice of the Green's function that vanishes at the plane integration surface (thus, removing the second, purely-defined term in the Kirchhoff formulation), we further modify the Green's function to make it identically zero at the curved lens surface S2L while using the original form at the planar freespace surface S2F.

In this way we obtain the approximation denoted as RS2 model, since the modified Green's function consists of two terms similar to the original RayleighSommerfeld formulation.

In explicit form, the RS2 representation of the wave field $U_{R S}$ at observation point $\mathrm{P}$ is as follows:

$$
U_{R S}^{(2)}(P)=\int_{S 2} U \frac{d G_{R S}^{(2)}}{d n} d s
$$

where the modified Green's function is

$$
G_{R S}^{(2)}=G\left(r_{P}, r_{S 2}\right)-G\left(r_{P}^{\prime}, r_{S 2}^{\prime}\right)
$$

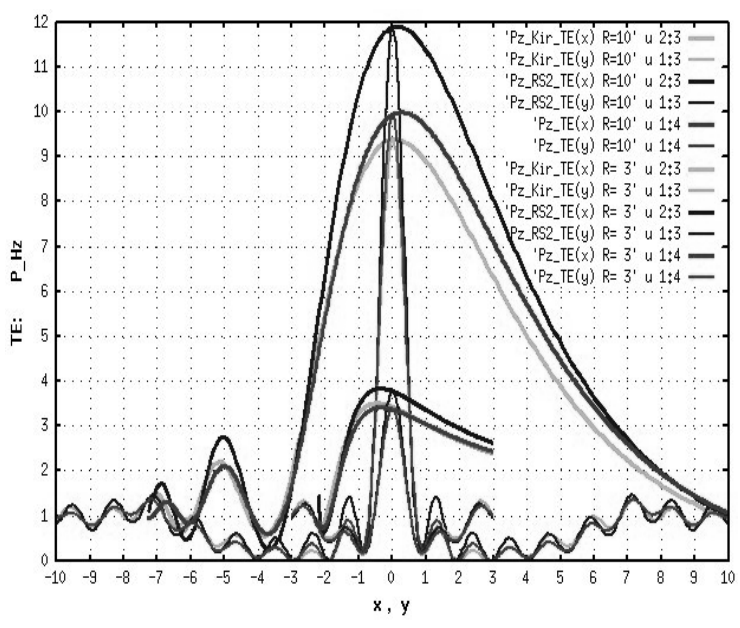

Fig. 3. KU and RS2 asymptotic methods in comparison to the exact solutions for symmetric lenses with $D=3 \lambda$ and $D=10 \lambda$ (TE waves, $n=1.5, f=R_{1}=R_{2}=D, \theta_{l}=\theta_{2}=30^{\circ}$ ).

with $r_{p}^{l}$ and $r_{s 2}^{l}$ being the image points of $r_{p}$ and $r_{s 2}$ with respect to the effective plane of the lens.

Notice, the RS2 model described above is superior to certain generalizations of the Rayleigh-Sommerfeld approach being proposed for curved surfaces as, e.g., those in [12] where no modification of the Green's function is made, or in [13] where an alternative fourterm Green's function is introduced which, however, generates entirely wrong results when tested in our simulations.

\subsection{COMPARISON OF EXACT AND ASYMPTOTIC WAVE-LIKE SOLUTIONS}

An example of the comparison of exact solutions with asymptotic wave-like approximations of KU and RS2 models is shown in Fig. 3. Here, the lens specified by the parameters $D \sim f>\lambda$ is represented.

Both the approximations are considered as being rather accurate, with maximum deviation from the exact results observed at the focal point at the level of about $10 \%$ to $20 \%$ for different models and lens parameters.

The deviations arise due to (a) neglect of the edge diffraction and the internal reflection in the lens, (b) inaccuracy of the ray model for the wave propagation to the lens surface S2L and (c) additional approximation in the KU model needed for the evaluation o the normal derivative of the complex field amplitude at the lens surface S2L.

Fig. 4 shows the power and phase patterns of the refracted wave focused behind the lens with the geometrical focal point $\mathrm{O} 1(x=y=0)$. The solution is obtained by using the RS2 asymptotic model.

\subsection{ASYMPTOTIC SOLUTIONS FOR LARGE AND SMALL-SCALE LENSES}

In practice, the asymptotic wave-like approximations would be particularly useful for large-scale lenses, 


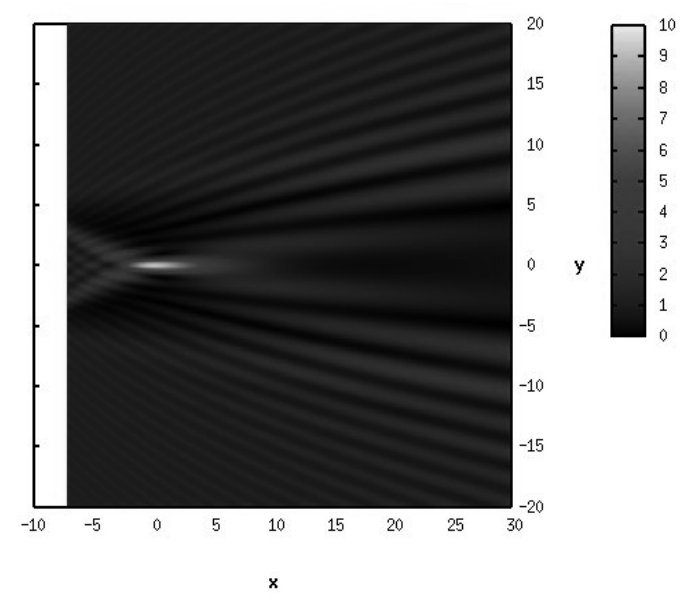

(a)

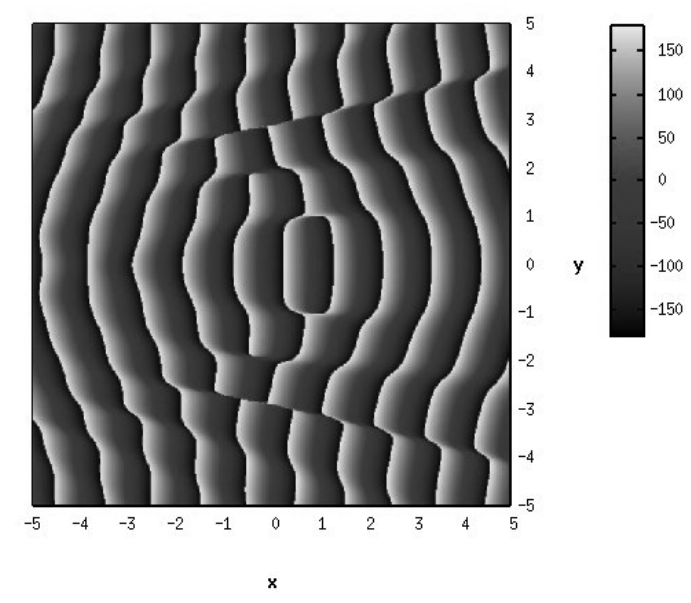

(b)

Fig. 4. (a) Power and (b) phase patterns of the TE wave in the focal domain of lens $D=10 \lambda$ in Fig. 3 (the patterns computed by using the RS2 asymptotic model).

especially, in three-dimensional problems, for which no exact solutions can realistically be obtained, while asymptotic methods are, typically, extremely efficient.

Fig. 5 shows the longitudinal distribution of $\mathrm{H}_{z}{ }^{2}$ power flux component (TE wave) in the focal domain of relatively large lenses $(n=1.5, D=100 \lambda$, and $f=$ $\left.R_{I}=R_{2}=\{100,150,400\} \lambda\right)$ computed with $\mathrm{KU}$ and RS2 methods $(f>D>>\lambda)$. One can see an excellent coincidence of results of two methods that improves with increasing the size of lens.

Another example is shown in Fig. 6 for the lens of small diameter (microlens, $D=\lambda$ ) and rather large focal length $\left(f=R_{1}=R_{2}=\{20,30,40\} \lambda\right)$. The coincidence of the results of KU and RS2 methods in this case is also excellent, with two curves superimposed precisely when $f=40 \lambda$.

\subsection{OPTIMAL ASYMPTOTIC APPROXIMATIONS FOR ASYMMETRIC LENSES}

The results above are obtained for symmetric lenses that have identical curvature radii $R I=R 2$ of the lens

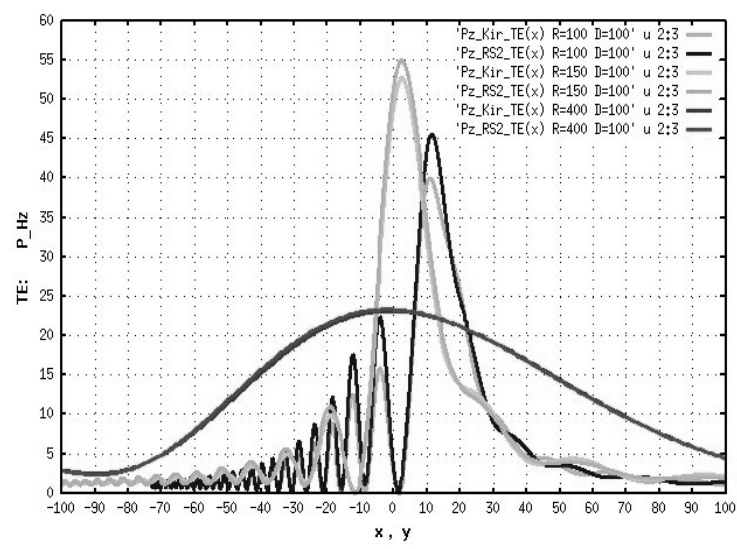

Fig. 5. $H_{z}^{2}$ power flux component (TE wave) in the focal domain of a rather large lens $(n=1.5$, $D=100 \lambda, f=R_{1}=R_{2}=\{100,150,400\} \lambda$, $\theta_{1}=\theta_{2}=30^{\circ}-7^{O}$ ) computed with $\mathrm{KU}$ and RS2 asymptotic methods $(f>D>>\lambda)$.

surfaces S1L and S2L, respectively. For asymmetric lenses with $R 1>>R 2$ and for microlenses of small focal length $(f \sim D \sim \lambda)$ we observe, however, certain problems. For these lenses, the results of two asymptotic methods differ significantly (Fig. 7).

Further analysis revealed that, in these cases, we observe the effect of total internal reflection of rays near the lens edges. Due to this effect, no wave can propagate through the lens near the rim (Fig. 8). Moreover, the area of the lens surface near the rim where the wave would experience total internal reflection appeared to be quite significant in many cases considered.

Even though both the KU and RS2 simulation models took the effect into account by assigning zero wave amplitude in this area, the inconsistency of the entire field distribution in this domain is too significant for the models to be reliable.

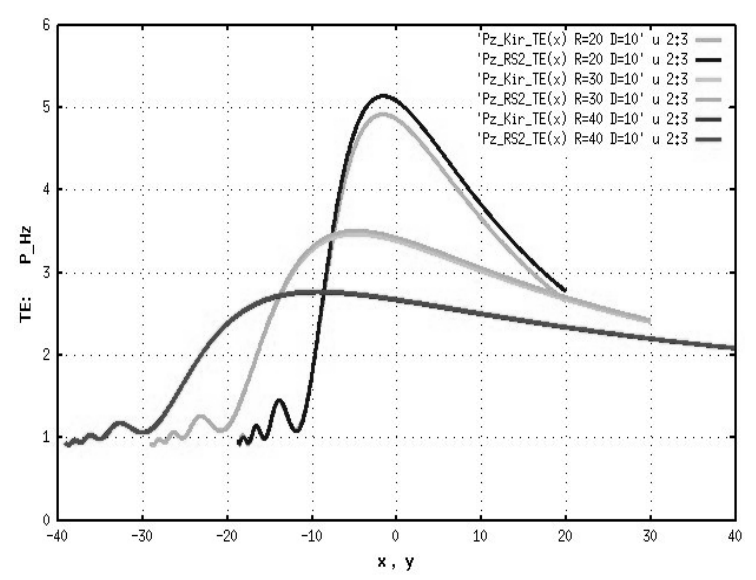

Fig. 6. $H_{z}^{2}$ power flux component (TE wave) in the focal domain of a microlens $(n=1.5, D=\lambda)$ with relatively large focal length $\left(f=R_{1}=\right.$ $\left.R_{2}=\{20,30,40\} \lambda, \theta_{1}=\theta_{2}=14^{O}-7^{O}\right)$ computed with KU and RS2 asymptotic methods $(f>>D \sim \lambda)$. 


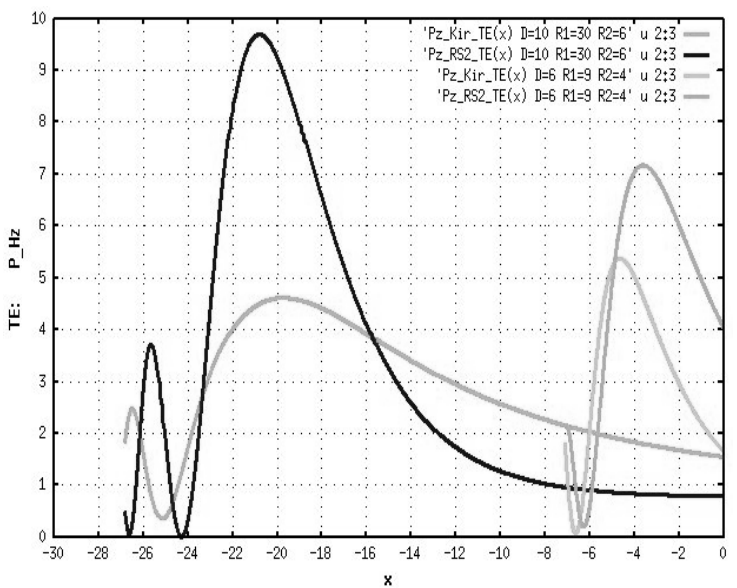

Fig. 7. $H_{z}^{2}$ power flux component (TE wave) in the focal domain of asymmetric lenses $(n=1.5$, $D=10 \lambda, R_{1}=30 \lambda, R_{2}=6 \lambda, \theta_{1}=9.6^{\circ}$, $\theta_{2}=56.4^{\circ}$ ) computed with KU and RS2 asymptotic methods.

In order to compare the relative applicability of different asymptotic models for asymmetric lenses, we generated an exact full-wave solution for certain special cases where the solution of this kind is accessible

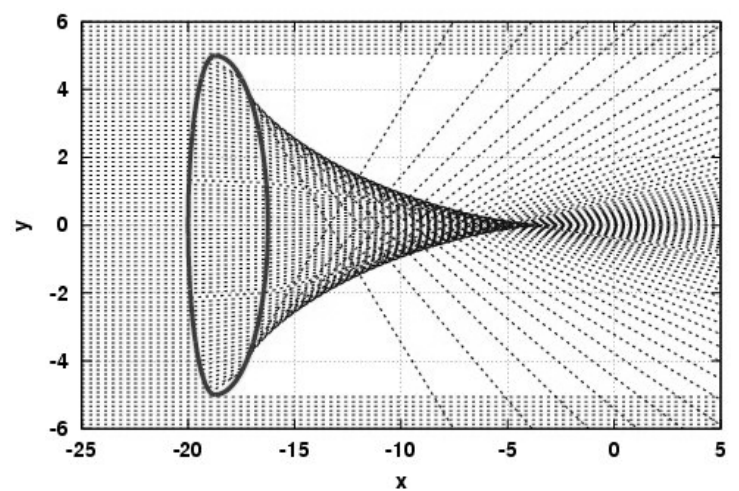

(a)

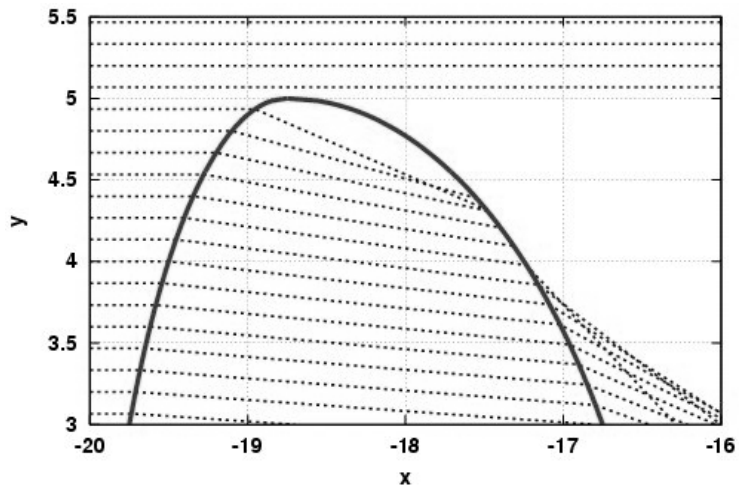

(b)

Fig. 8. Total internal reflection near the lens rim as illustrated by ray tracing for the model case of asymmetric 2D lens with elliptical surfaces (six rays near the rim in this illustration do not propagate through the lens).

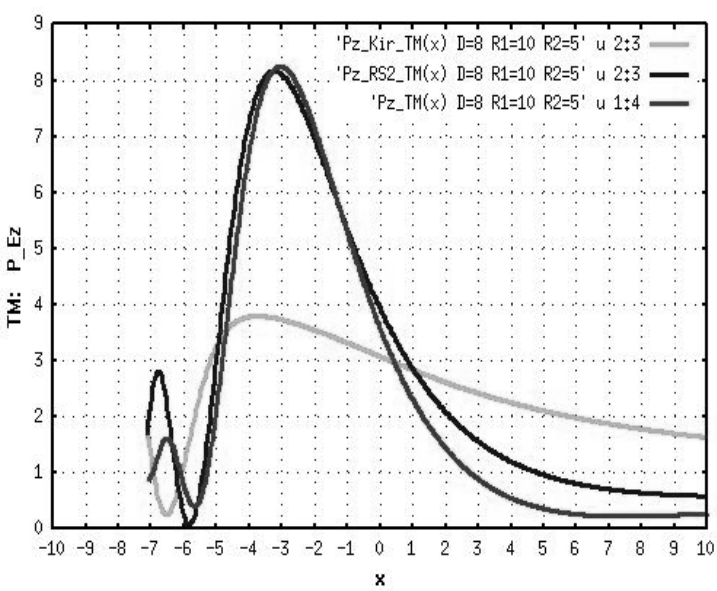

Fig. 9. KU and RS2 asymptotic methods in comparison to the exact solution (TM wave) for the asymmetric lens with $D=8 \lambda, R_{1}=10 \lambda$, $R_{2}=5 \lambda\left(\theta_{1}=23.6^{O}, \theta_{2}=53.1^{O}, n=1.5\right)$. The RS2 approximation is very close to the exact solution whereas KU model fails crucially.

(Fig. 9). It appears, remarkably, that exact solutions are also quite difficult to obtain by direct methods precisely in those cases when the effect of total internal reflection is observed.

The comparison of asymptotic and exact solutions obtained for relatively large asymmetric lenses with the curvature radii R1 $>$ R2 has shown that, generally, the Rayleigh-Sommerfeld model RS2 based on the modified Green's function for curved surfaces has an advantage over the Kirchhoff formulation KU with approximation of the normal derivative of the complex wave amplitude at the exit lens surface.

In all the cases considered, the RS2 method proved to be capable of relatively accurate representation of the wave field in the focal domain of asymmetric lenses as compared to the exact solution, whereas the $\mathrm{KU}$ method crucially failed in these circumstances.

\section{Conclusions}

In this work, we proposed and analyzed a few asymptotic wave-like approximations for the accurate and efficient modeling of dielectric lenses used in quasioptical systems of millimeter, submillimeter and infrared wave applications. For the comparison, we developed an exact full-wave analytic solution of twodimensional focusing lens problem and used it as a benchmark for testing and validation of asymptotic models being proposed.

The main asymptotic formulations considered are the two-term Kirchhoff model with an appropriate approximation of the normal derivative of complex wave amplitude at the lens surface (KU) and the oneterm Rayleigh-Sommerfeld diffraction integral formulation modified for non-planar (curved) exit lens surfaces (RS2). 
The Rayleigh-Sommerfeld approximation modified for curved surfaces (RS2 model) appeared to be more general and better suited for various kinds of dielectric lenses, including symmetric and asymmetric, thin and thick, rather large and relatively small lenses.

Both the Kirchhoff model (KU) and the RayleighSommerfeld representation modified for non-planar surfaces (RS2) are remarkably accurate for large lenses $(f, D>>\lambda)$ where no total internal reflection effects occur (i.e., typically, for symmetric lenses or sufficiently thin asymmetric ones with relatively flat exit surface).

Both the KU and RS2 approximations are also surprisingly accurate for small lenses, including the microlenses, when both the lens diameter $D$ and the focal length $f$ are comparable with the radiation wavelength $\lambda(f \sim D \sim \lambda)$, though small lenses have to be symmetric for minimizing the possibility of total internal reflection effects.

The KU model fails, however, for bulk asymmetric lenses with relatively convex exit surface where the total internal reflection occurs for the incident waves near the lens rim.

\section{ACKNOWLEDGMENTS}

This work was supported, in part, by Enterprise Ireland through a Basic Research Grant.

The authors are grateful to J. A. Murphy for useful discussions.

\section{REFERENCES}

1. Bowden M. et al., "Measuring the cosmic microwave background polarization with the QUaD experiment," Proc. SPIE, vol. 5489, 84-94 (2004)

2. Church S. et al., "QUEST on DASI: a South Pole CMB polarization experiment," New Astronomy Reviews, vol. 47, 1083-1089 (2003)

3. Balanis C.A., Advanced Engineering Electromagnetics, John Wiley and Sons, New York, 1989
4. Sadiku, M.N.O., Numerical Techniques in Electromagnetics, CRC Press, London, 1992

5. Muller C., Foundations of the Mathematical Theory of Electromagnetic Waves, Springer-Verlag, Berlin, 1969.

6. Prather D.W., Mirotznik M.S., and Mait J.N., "Boundary integral methods applied to the analysis of diffractive optical elements," J. Opt. Soc. Am. A, vol.14, 34-43 (1997)

7. Fikioris G., "A note on the method of analytical regularization," IEEE Antennas and Propagation Magazine, vol. 43, 34-40 (2001)

8. Boriskina S.V. et al., "Accurate simulation of twodimensional optical microcavities with uniquely solvable boundary integral equations and trigonometric Galerkin discretization," J. Opt. Soc. Am. $A$, vol. 21, 393-402 (2004)

9. Boriskin A.V. et al., "Lens or resonator? Electromagnetic behavior of an extended hemielliptic lens for a sub-millimeter-wave receiver," Microwave and Opt. Technol. Letters, vol. 43, 515-518 (2004)

10. Ye J.S. et al., "Analysis of a closed-boundary axilens with long focal depth and high transverse resolution based on rigorous electromagnetic theory," J. Opt. Soc. Am. A, vol. 19, 2030-2035 (2002)

11. Feng D. et al., "Axial focusing characteristics of diffractive micro-lenses based on a rigorous electromagnetic theory," J. Opt. A Pure Appl. Opt. vol. 6, 1067-1071 (2004)

12. Ye J.S. et al., "Application of improved first Rayleigh-Sommerfeld method to analyze the performance of cylindrical microlenses with different f-numbers," J. Opt. Soc. Am. A, vol. 22, 862-869 (2005)

13. Duan K. et al., "Improved diffraction integral for studying the diffracted field of a spherical microlens," J. Opt. Soc. Am. A, vol. 22, 2677-2681 (2005) 